\title{
Analysis of Interference \& BER with Simulation Concept for MC-CDMA
}

\author{
Ravisha ${ }^{1}$, Mrs. Saroj ${ }^{2}$ \\ M.Tech.Scholar, ${ }^{1}$ Asst. Professor ${ }^{2}$ \\ Electronics and Communication Department CBS Group of Institution, Jhajjar Haryana
}

\begin{abstract}
As demand for higher data rates is continuously rising, there is always a need to develop more efficient wireless communication systems. Efficient use of the spectrum is an essential problem in creating any multi-user cellular mobile system. The bandwidth requirements of the future $4 G$ systems far exceed what the existing traditional setup can provide. A more creative utilization of the available bandwidth is more important now than ever before. The work described in this paper is my effort in this direction. I evaluated interference and bit error rate for multicarrier code division multiple access wireless communication system. In this thesis my concern is find out the effect of fading in MC DS-CDMA system. Performance analysis of a multi carrier direct sequence CDMA system will be carried out including the effect of fading. In this Paper, our main objective is to evaluate the effect of fading and Inter Carrier Interference (ICI) in a MC DS CDMA wireless system, to find out the expression of Unconditional BER of MC DS CDMA system including the above system limitations. I find out the analytical results of interference and BER analysis. Simulations are given to support the system and receiver design. All the simulation is carried out on MATLAB tool.
\end{abstract}

\section{Introduction}

Multicarrier modulation is a data transmission technique where several sub carriers are employed to transport the user's data stream signal. Today using very fast and cost effective digital signal processors, multicarrier modulation can be implemented using discrete signal processors; multicarrier modulation can be implemented using discrete DFT as the set of orthogonal sub carrier. This makes the technique very attractive. Multicarrier modulation improves system capacity by making transmission more robust to frequency selective fading and enhances user spectral efficiency. The basic idea to use multicarrier transmission in a CDMA system is to extend the symbol duration so that a frequency-selective fading channel is divided into a number of narrow band flat fading channels, and the complex time-domain equalization can therefore be replaced with a relatively simple frequency domain combining.

Normally an Inverse Fast Fourier Transform (IFFT) block is used in the transmitter to modulate user data in the sub carriers and an FFT block is used in the receiver to demodulate the data so as to achieve fast computation. Frequency-domain diversity can be easily achieved in multicarrier CDMA systems by means of frequency diversity schemes. However, sinusoid waveforms are not well localized in the time domain. Thus, time-domain diversity within one symbol period is difficult to achieve. Therefore, a guard interval is needed to eliminate residual intersymbol interference (ISI) due to multipath. This method requires transmitting extra guard interval signals, which introduces overhead and thus decreases bandwidth efficiency.

\section{Mc-Cdma}

The combination of multicarrier transmission and CDMA can be achieved in different ways. Consequently, the multiplexing CDMA designs fall in two categories:

(a) Frequency Domain Spreading

MC-CDMA combines the multicarrier transmission with the frequency domain spreading, i.e., the original data stream from a user is spread with this user's specific spreading code in the frequency domain but not in the time domain. In other words, each symbol is transmitted simultaneously in a number of subcarriers, but multiplied by corresponding chips of the spreading code for every subcarrier Fig.1 and Fig.2 give the transmitter and receiver structures of an MC-CDMA. It can be seen that the data rate for each subcarrier is only $1 / \mathrm{N}$ as that of a single carrier DS-CDMA system. This means that the chip duration is $\mathrm{N}$ times longer. Therefore, the channel delay spread is comparatively shorter. If it is much shorter than the extended chip duration, the original frequency selective fading channel is divided into a number of flat fading channels. Thus, the complicated time domain equalization can be replaced by a simple gain combining in the frequency domain. 


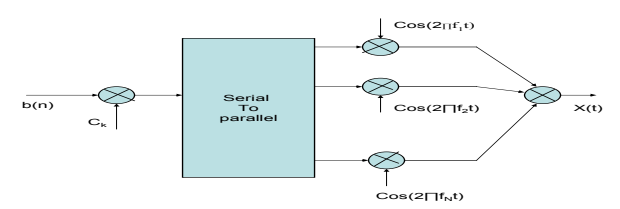

Fig 1 MC-CDMA Transmitter

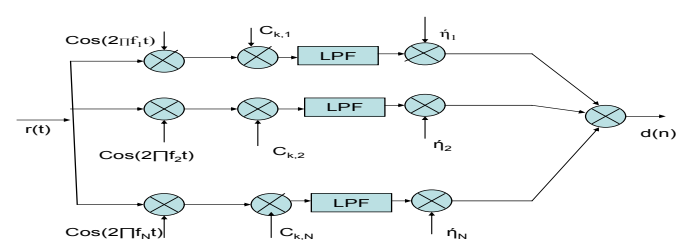

Fig 2MC-CDMA Receiver

(b) Time Domain Spreading

Another way of combining multicarrier modulation with CDMA is the MC -DS-CDMA scheme that spreads the original user data stream in the time domain. As shown in Fig.3, the user data stream is first serial to parallel converted into $\mathrm{N}_{\mathrm{c}}$ (the number of subcarriers) sub streams, each of which is time-spread and transmitted in an individual subcarrier. In other words, a block of $\mathrm{N}_{\mathrm{c}}$ symbols are transmitted simultaneously. The value of $\mathrm{N}_{\mathrm{c}}$ can be chosen according to the system design requirement. However, it is commonly assumed to be equal to the length of spreading code $\mathrm{N}$ which will also make the comparison with MC-CDMA easier .All the symbols are spread in the time domain using the same spreading code for a particular user. It is clear that this scheme achieves time domain diversity but no frequency domain diversity for each individual data symbols. The sub carriers satisfy the same orthogonality condition as that of MC-CDMA. This scheme is suitable for uplink transmission since it is easy for the establishment of quasisynchronization between different users. Fig.4 gives the basic structure of the receiver of the MC -DSCDMA system where each branch equals to a single CDMA signal detector.

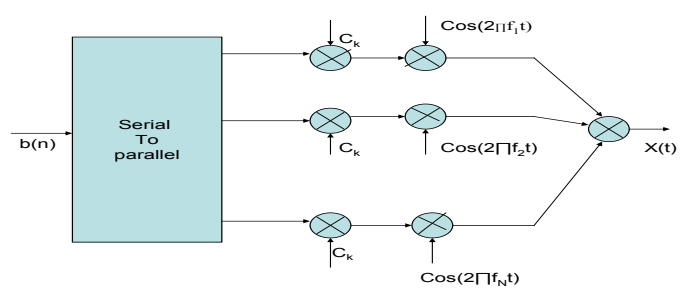

Fig 3 MC-DS-CDMA Transmitter

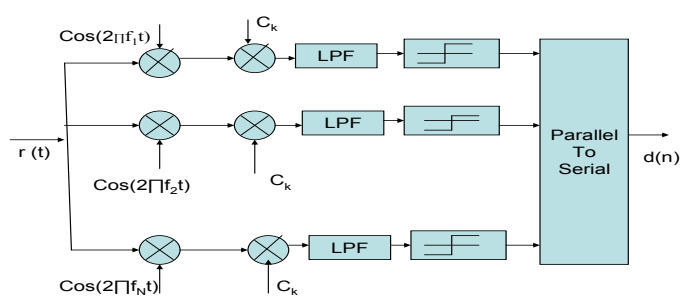

Fig 4 MC-DS-CDMA Receiver

Another time domain spreading multicarrier CDMA scheme called MT-CDMA scheme uses much longer spreading codes so that the bandwidth for each subcarrier signal is about the same as the original DS-CDMA signal. The signals for different subcarriers overlap heavily and do not satisfy orthogonality condition, but longer spreading codes help to eliminate the multi-user interference . 


\section{Mc-Cdma Transmitter Model}

The MC-CDMA transmitter configuration for the $\mathrm{j}^{\text {th }}$ user is shown in Fig 5.

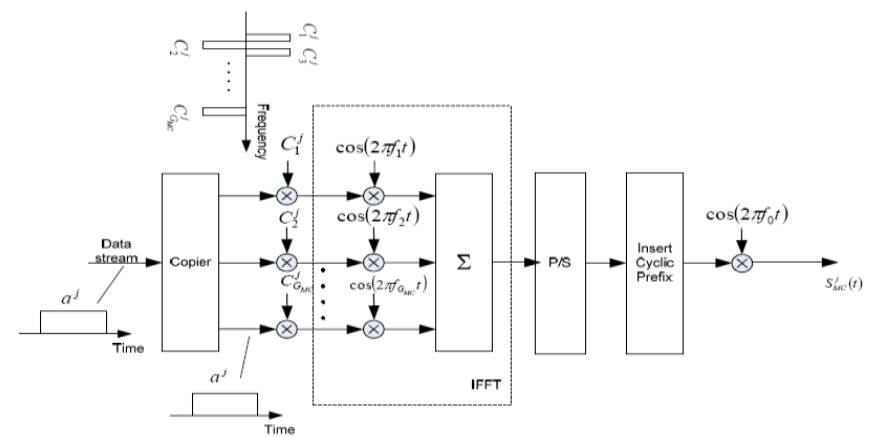

Fig 5 MC-CDMA transmitter

In this figure, the main difference is that the MC-CDMA scheme transmits the same symbol in parallel through several subcarriers whereas the OFDM scheme transmits different symbols. $C_{j}(t)=\left[\begin{array}{llll}C_{1}^{j} & C_{2}^{j} & \cdots & C_{G_{M C}}^{j}\end{array}\right]$ is the spreading code of the $\mathrm{j}^{\text {th }}$ user in the frequency domain, $\mathrm{G}_{\mathrm{MC}}$ denotes the processing gain, sometimes called the spreading factor. The input data stream is multiplied by the spreading code with length $\mathrm{G}_{\mathrm{MC}}$. Each chip of the code modulates one subcarrier. The number of subcarriers is $N=\mathrm{G}_{\mathrm{MC}}$ The users are separated by different codes. All data corresponding to the total number of subcarriers are modulated in baseband by an inverse fast Fourier transform (IFFT) and converted back into serial data. Then, a cyclic prefix is inserted between the symbols to combat the inter-symbol interference (ISI) and the inter-carrier interference (ICI) caused by multipath fading. Finally, the signal is digital to analog converted and upconverted for transmission.

In MC-CDMA transmission, it is essential to have frequency nonselective fading over each subcarrier. Therefore, if the original symbol rate is high enough to become subject to frequency selective fading [1], the input data have to be S/P converted into $P$ parallel data sequences $\left[\begin{array}{llll}a_{1}^{j} & a_{2}^{j} & \cdots & a_{P}^{j}\end{array}\right]$ and each S/P output is multiplied with the spreading code of length $\mathrm{G}_{\mathrm{MC}}$. Then, each sequence is modulated using $\mathrm{G}_{\mathrm{MC}}$ subcarriers. Thus, all $N=P \times \mathrm{G}_{\mathrm{MC}}$ subcarriers (total data) are also modulated in baseband by the IFFT.

\section{Mc-Cdmareceiver Model}

The MC-CDMA receiver configuration for the $j t h$ user is shown in Fig 6. The received signal is first down converted. Then, the cyclic prefix is removed and the remaining samples are serial to parallel converted to obtain the $m$-subcarriers components (corresponding to the $\mathrm{a}_{\mathrm{j}}^{\mathrm{p}}$ data), where $m=1,2, \ldots \ldots \ldots . \mathrm{G}_{\mathrm{MC}}$

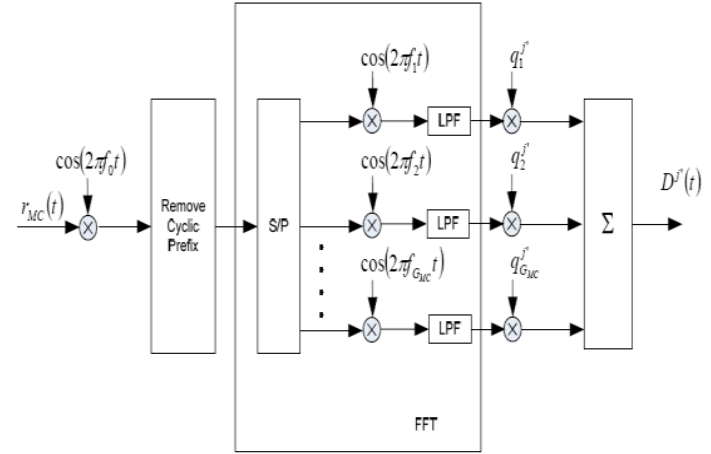

Fig 6 MC-CDMA receiver

The $m$-subcarriers are first demodulated by a fast Fourier transform (FFT) (OFDM demodulation) and then multiplied by the gain $\mathrm{q}_{\mathrm{m}} \mathrm{j}^{\prime}$ to combine the received signal energy scattered in the frequency domain. The decision variable is given by

$$
D^{j^{\prime}}=\sum_{m=1}^{G_{M C}} q_{m} y_{m}
$$

And 


$$
y_{m}=\sum_{j=1}^{J} z_{m}^{i} a^{j} c_{m}^{j}+n_{m}
$$

where $y_{m}$ and $n_{m}$ are the complex baseband component of the received signal and the complex Gaussian noise at the $\mathrm{m}^{\text {th }}$ subcarrier, respectively. $z_{m}{ }^{j}$ and $a^{j}$ are the complex envelope of the $m^{\text {th }}$ subcarrier and the transmitted symbol of $\mathrm{j}^{\text {th }}$ user, respectively. $\mathrm{J}$ is the number of active users.

(i)

\section{Simulation Result}

The number of user with Inter Carrier Interference :

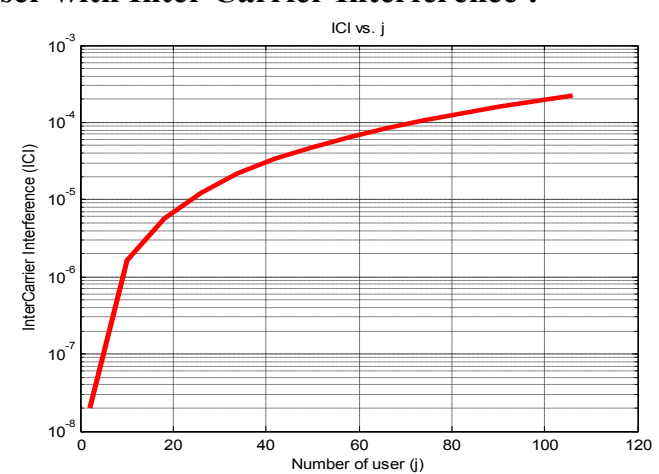

Fig 7 ICI vs. $\mathbf{j}$

Here we can see that the plot is about the number of user (j) vs. Inter Carrier Interference (ICI). If we increase the number of user (j) then the inter carrier interference (ICI) will be increase. Here the number of user is 14 times, signal power is $1 \mathrm{mw}$ and the we have used the ICI equation and the curve is for the value of alpha= 0.2

\section{(ii) The signal power (Ps) with bit error rate in $\mathrm{db}$}

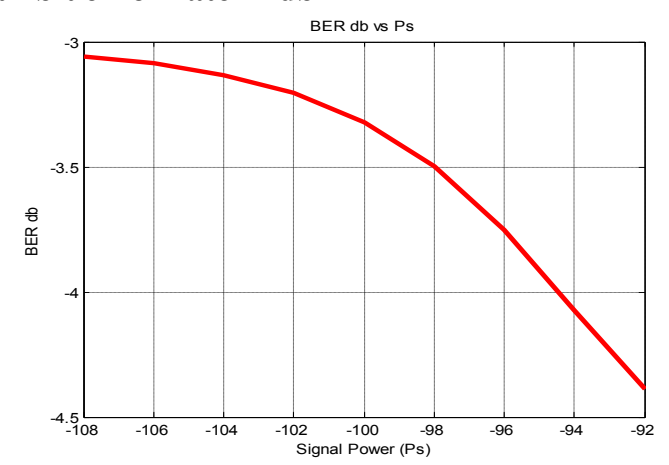

Fig 8 BER db vs. Ps

Here we can see that the plot is in between the signal power (Ps) vs. bit error rate in $\mathrm{db}$ form. If we increase the signal power $(\mathrm{Ps})$ then bit error rate $(\mathrm{BER} \mathrm{db})$ will be decrease. Here for alpha $=0.2$ we got the curve for 10 user and four sub-carrier.

(iii) The numbers of user ( $\mathrm{Nu}$ ) with unconditional Bit error rate (BER)

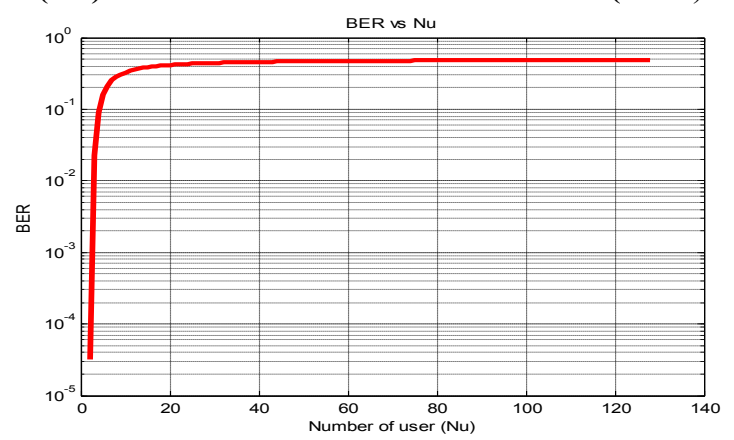

Fig 9 BER vs. Nu 
Here, we can see the plot between the numbers of user $(\mathrm{Nu})$ with unconditional Bit error rate (BER). If we increase the number of user $(\mathrm{Nu})$ the bit error rate (BER) will be increase. After reach in nee point the bit error rate is getting fixed. We have used the formula of unconditional bit error rate. Here the number of user $(\mathrm{Nu})$ is 128 and each user divided into 1000 .

The Number of user $(\mathrm{Nu})$ with unconditional Bit error rate $(\mathrm{BER})$ with $\mathrm{Nc}=32,64,128,200$

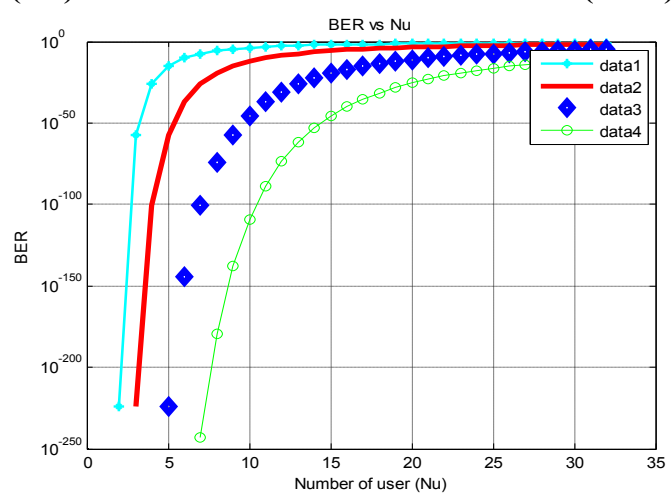

Fig 10 BER vs. Nu (with value of $\mathrm{Nc}$ )

Here we can also see that the plot between the Number of user $(\mathrm{Nu})$ vs. unconditional Bit error rate (BER). In this plot, we consider the number of sub-carrier $(\mathrm{Nc})$ and vary this into different values such as $\mathrm{Nc}=$ $32,64,128,200$.

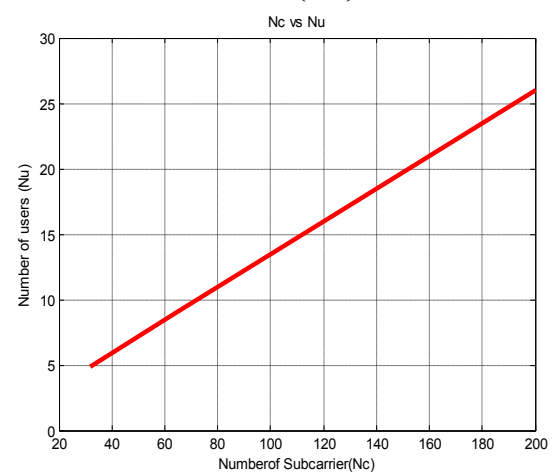

Fig 11 Nu vs. Ne

This plot is from the previous graph. For different values of $\mathrm{Nu}$ we got different values of $\mathrm{Nc}$. Here the conditional bit error rate is $10^{\wedge}-15$. If we increase the number of sub-carrier $(\mathrm{Nc})$ then the number of users $(\mathrm{Nu})$ will be also be increase.

\section{Conclusion}

This paper reviews Multicarrier based CDMA schemes such as MC CDMA, DS CDMA and MC DS CDMA with the effect of fading and discuss about their some features. This paper is basically focused on the MC DS CDMA system and its analysis with fading. In this paper I use MATLAB to find some plots to verify the concept of multicarrier CDMA and finally I concluded the following facts that On comparing the numbers of user (j) with inter-carrier interference (ICI)as we increase the number of user (j) then the inter carrier interference (ICI) will be increase. And on comparing BER db with signal power (Ps) as if we increase the signal power (Ps) then bit error rate will be decreasing. Also on comparing the Number of user (Nu) with unconditional Bit error rate (BER) as we increase the number of user $(\mathrm{Nu})$ the bit error rate (BER) will be increase. On comparing the Number of user $(\mathrm{Nu})$ with unconditional Bit error rate (BER) we conclude that with the increase the number of user $(\mathrm{Nu})$ the bit error rate (BER) will be increase and finally on comparing the number of sub-carrier $(\mathrm{Nc})$ with the number of users $(\mathrm{Nu})$ as we increase the number of sub-carrier $(\mathrm{Nc})$ then the number of users $(\mathrm{Nu})$ will be also be increase. 


\section{References}

[1] V.Nagarajan and P. Dananjayan, "Performance Analysis of MIMO MC-DS/CDMA System Using Chaotic Spreading Sequence" , International Journal of Computer and Electrical Engineering, Vol. 2, No. 2, April, 2010

[2] Tao LUO, Gang WU, and Shaoqian LI, Yong Liang GUAN, Choi Look LAW, "DS-CDMA and MC-CDMA with Per-User MMSE Frequency Domain Equalization", International journal of hybrid information technology, vol 1.1,No. 3, july2008

[3] V.Nagarajan and P.Dananjayan, "Performance Enhancement Of Mc-Ds/Cdma System Using Chaotic Spreading Sequence" , Journal of Theoretical and Applied Information Technology, 2005 - 2008 JATIT.

[4] Salih M. Salih, N. Uzunoglu, A. A. Ali and Waleed A. Mahmud "A Proposed Improvement Model of MC-CDMA Based FFT in AWGN Channel” Pg.517-520, IEEE 2007.

[5] Shinsuke Hara and Ramjee Prasad, “Overview of Multicarrier CDMA”, Pg.126-133, IEEE Dec 1997.

[6] John G. Proakis, "Digital Communications". Mc. Graw Hill International, Fourth edition.

[7] S. Salivahanan, A. Vallavraj and C. Gnanapriya, "Digital Signal Processing", Tata Mc. Graw Hill Publishing Company Limited. 